\section{Genetic mutations and cryptorchidism}

Cryptorchidism is the most common congenital birth defect in boys. Testicular descent is regulated by testosterone and insulin-like factor 3 (INSL3). An Italian study investigated whether genetic defects affecting the function of these hormones were associated with cryptorchidism. The researchers found genetic defects in 5-8\% of boys with persistent and/or bilateral cryptorchidism.

Ferlin et al. followed up 600 newborn boys with cryptorchidism for 2-3 years. Mutation analysis of the INSL3, RXFP2 (relaxin family peptide 2, the receptor for INSL3) and $A R$ (androgen receptor) genes was performed; 300 boys without cryptorchidism were recruited as controls. Spontaneous descent occurred in 199 of 396 unilateral cases and 83 of 204 bilateral cases in the first 6 months of age; a further 14 unilateral cases and 1 bilateral case experienced spontaneous descent within 12 months. There were no instances of spontaneous descent after 12 months. Overall, 17 genetic abnormalities were found in the 600 boys with cryptorchidism. The rate of genetic abnormalities was $5.3 \%$ (16 of 303) in boys with persistent and $8.3 \%$ (10 of 120) in those with bilateral cryptorchidism, compared with $0.3 \%$ (1 of 300 ) in the control group. Eight of the defects discovered were chromosomal abnormalities of the Klinefelter type. Five boys with bilateral cryptorchidism had mutations in RXFP2, and there were two cases each of mutations in INSL3 and AR.

In a small percentage of the study population, there was a statistically significant association between bilateral and persistent cryptorchidism and genetic alterations, including Klinefelter syndrome and INSL3 receptor gene mutations.

Original article Ferlin A et al. (2008) Genetic alterations associated with cryptorchidism. JAMA 300: 2271-2276

\section{Linear mixed model helps to quantify PSA profile and predict disease progression}

Assessment of prostate-specific antigen (PSA) kinetics is often used to predict outcome after radiation therapy for localized prostate cancer. The decision to start salvage therapy is usually made before the development of symptomatic disease, and which PSA characteristics should be used for treatment decisions remains unclear. Proust-Lima and colleagues have developed an approach based on the relationships between prognostic factors, postradiotherapy PSA kinetics and clinical recurrence to provide an accurate picture of the risk of recurrence.

The authors analyzed 40,324 PSA measurements from 4,247 patients treated with radiation therapy. A linear mixed model was used to describe the pattern of PSA changes after radiotherapy. A Cox model was then used to determine the association between prognostic factors, post-treatment PSA behavior and time to recurrence.

Clinical recurrences were observed in $12.2 \%$ of the patients. Short-term declines in PSA levels after radiation were significantly associated with pretreatment PSA and tumor stage. High values of pretreatment PSA, tumorstage and Gleason score were associated with steep subsequent increases in PSA. A high current PSA level and steep rate of PSA increase were both associated with a high risk of recurrence.

The authors conclude that a joint linear mixed model can be used to assess the PSA kinetics and risk of recurrence after radiation therapy for prostate cancer and have developed a risk calculator for others to use.

Original article Proust-Lima C et al. (2008) Determinants of change in prostate-specific antigen over time and its association with recurrence after external beam radiation therapy for prostate cancer in five large cohorts. Int $J$ Radiat Oncol Biol Phys 72: 782-791 